\title{
Synergistic effects of bevacizumab in combination with $\beta$-elemene on subcutaneous xenografts derived from HCT-116 human colon cancer cells
}

\author{
Jin-Na Li, Feng Xie, Ying Wang \\ Department of Oncology, Shengjing Hospital Affiliated to China Medical University, Shenyang 110000, China \\ Contributions: (I) Conception and design: JN Li, Y Wang; (II) Administrative support: Y Wang; (III) Provision of study materials or patients: Y Wang; \\ (IV) Collection and assembly of data: JN Li, F Xie; (V) Data analysis and interpretation: F Xie, JN Li; (VI) Manuscript writing: All authors; \\ (VII) Final approval of manuscript: All authors. \\ Correspondence to: Ying Wang. Professor, Department of Oncology, Shengjing Hospital Affiliated to China Medical University, 36 Sanhao Street, \\ Shenyang 110000, China. Email: wang_ying@sj-hospital.org.
}

Background: To observe the effect of bevacizumab in combination with $\beta$-elemene on subcutaneous xenografts established from HCT-116 human colon cancer cells, explore the underlying mechanisms involved, and provide more options for the treatment of advanced colorectal cancer.

Methods: Mice were randomly divided into four groups: control, $\beta$-elemene, bevacizumab and combination treatment. Tumor volume was measured and the tumor inhibition rate and coefficient of drug interaction (CDI) were calculated. The pathological and morphological features of the tumor tissues were observed. Protein expression levels of ki-67, CD31, vascular endothelial growth factor (VEGF) and bcl-2 were measured by immunohistochemistry.

Results: The tumor volumes in the four groups were $0.991 \pm 0.131,0.833 \pm 0.145,0.563 \pm 0.131$ and $0.324 \pm 0.066 \mathrm{~cm}^{3}$, respectively. The tumor inhibition rates in the $\beta$-elemene, bevacizumab and combination groups were $15.9 \%, 43.2 \%$ and $67.3 \%$, respectively. The degree of tumor reduction in the combination group was the most significant $(\mathrm{P}<0.05)$, with a CDI of 0.697 . Immunohistochemistry showed that the ki-67 proliferation indexes in the four groups were $30.06 \% \pm 3.37 \%, 19.79 \% \pm 3.75 \%, 27.51 \% \pm 2.16 \%$ and $16.9 \% \pm 1.09 \%$, the microvessel densities (MVDs) were 45.86 $\pm 9.28,31.14 \pm 5.58,27.57 \pm 6.11$ and $12.14 \pm 3.53$, the rates of positive VEGF expression were $39.48 \% \pm 6.72 \%, 28.86 \% \pm 3.54 \%, 25.04 \% \pm 2.64 \%$ and $19.5 \% \pm 2.68 \%$, and the rates of positive bcl-2 expression were $33.95 \% \pm 6.38 \%, 20.11 \% \pm 9.49 \%$, $24.28 \% \pm 6.57 \%$ and $11.18 \% \pm 2.72 \%$, respectively. Compared with the control group, the three experimental groups displayed different degrees of reduction in the above indicators $(\mathrm{P}<0.05)$, with the combination group exhibiting the lowest values $(\mathrm{P}<0.05)$.

Conclusions: Bevacizumab exerts a synergistic effect with $\beta$-elemene in suppressing the growth of tumors derived from HCT-116 cells, and the related mechanisms may include the inhibition of tumor cell proliferation and tumor angiogenesis and the promotion of tumor cell apoptosis.

Keywords: Bevacizumab; $\beta$-elemene; advanced colorectal cancer; apoptosis; angiogenesis; synergistic effect

Submitted Aug 20, 2019. Accepted for publication Nov 29, 2019.

doi: $10.21037 /$ tcr.2019.12.35

View this article at: http://dx.doi.org/10.21037/tcr.2019.12.35 


\section{Introduction}

Colorectal cancer, one of the most common malignancies, is the third most frequently diagnosed cancer, ranking as the third and second highest cause of cancer-related mortality in females and males, respectively, worldwide (1). Nearly half of the patients can be diagnosed by early detection methods and cured by radical surgery. Nevertheless, some patients already present metastasis at the time of diagnosis, or relapse after surgical treatment. For these patients with unresectable metastatic colorectal cancer, chemotherapy based on fluorouracil, oxaliplatin and irinotecan in combination with targeted therapies such as bevacizumab or cetuximab is the standard first-line treatment regimen (2). However, some patients cannot tolerate standard treatment due to its side effects, its toxicity or drug resistance. Therefore, the search for antitumor drugs with high efficacy and low toxicity has become a new research focus.

Neoangiogenesis is a key factor in tumor growth. New blood vessels provide not only oxygen and nutrients for tumor cells but also paths for the tumor cells to enter the circulation (3). Folkman found that the processes of tumor growth, invasion and metastasis depended on angiogenesis, and proposed the hypothesis underlying antiangiogenesis therapy in 1971. Antiangiogenesis therapy is achieved by inhibiting the formation of blood vessels and blocking the supply of nutrients and oxygen to tumor cells to curb tumor growth and metastasis (4). In addition, tumor growth is related to the imbalance between apoptosis and proliferation, and depends on tumor cells and vascular endothelial cells, which are interdependent. Endothelial cells in new blood vessels stimulate tumor cells proliferation by expressing various growth factors, and tumor cells secrete a variety of substances to promote the formation of new blood vessels during the process of tumor growth (5). Thus, direct inhibition of tumor cell proliferation, promotion of apoptosis or administration of antiangiogenesis therapy results in antitumor effects. Furthermore, combination treatments may be more effective for tumor inhibition, because the two drugs can act synergistically.

Tumor cells secrete vascular endothelial growth factor (VEGF)-A, a growth factor that results in neoangiogenesis by binding its tyrosine kinase receptors, VEGF receptors (VEGFRs), which are located on the surface of epithelial cells. An increase in angiogenesis facilitates blood flow to malignant cells, contributing to their growth and dissemination by ensuring a supply of oxygen and nutrients. Bevacizumab is a kind of recombinant human monoclonal IgG1 antibody, that interacts with VEGF-A to form a new molecule that lacks the ability to bind VEGFRs. This restriction inhibits new vessel formation and stabilizes existing tumor blood supply (6). On the one hand, this strategy reduces the blood supply to tumor tissues through antiangiogenesis, thereby promoting the apoptosis of tumor cells. On the other hand, it can reduce the vessel diameter, vascular basement membrane thickness, vascular permeability, and interstitial hydraulic pressure and increase vascular adventitial cell coverage to stabilize tumor blood vessels, which can maintain tumor cells in a highly proliferative state and increase their sensitivity to chemotherapy $(7,8)$. Compared with conventional chemotherapy, the combination of bevacizumab with chemotherapy as the first- and second-line treatment strategies for advanced colorectal cancer can prolong survival time by $30 \%$ (9). In addition, bevacizumab can be employed as a maintenance treatment for advanced colorectal cancer patients. However, the use of bevacizumab alone has limited efficacy, and bevacizumab needs to be combined with effective agents to elicit a response.

$\beta$-elemene is an antitumor drug extracted from a traditional Chinese medicinal herb Curcuma wenyujin, which has been approved by the State Food and Drug Administration of China as a second-class innovative drug (10). It is considered to exert a strong inhibitory and cytotoxic effect on tumor cells as a kind of traditional Chinese medicinal compound as shown in the present study, and it has been classified into the category of plant-based chemotherapeutic drugs. $\beta$-elemene is the active component of Curcuma wenyujin with the molecular formula C15H24 and molecular weight of 204.34 (11). Related studies have shown that $\beta$-elemene exerts antitumor effects by inhibiting tumor cell proliferation $(12,13)$, inducing apoptosis $(11,14)$, and suppressing angiogenesis $(15)$, among other functions. Studies confirm that $\beta$-elemene inhibits DNA and RNA synthesis in tumor cells, and arrests cells in $S$ phase to prevent them from entering $\mathrm{G}_{2} / \mathrm{M}$ phase (16). $\beta$-elemene downregulates the expression of antiapoptotic factors such as bcl-2, and bcl-xL, and upregulates the expression of proapoptotic factors such as bax, Fas, FasL and Caspase family members. $\beta$-elemene can also enhance the expression of the cancer suppressor gene P53 to induce apoptosis $(11,16)$. $\beta$-elemene combined with an IL-23transfected dendritic cell vaccine was shown to induce Th1 and cytotoxic T-lymphocyte (CTL) immune responses (17). $\beta$-elemene suppresses the proliferation endothelial cells and the expression of VEGF, basic fibroblast growth factor 
(bFGF) and other angiogenesis factors to play a role in antiangiogenesis (15).

Bevacizumab needs to be combined with effective chemotherapy agents, as the use of bevacizumab alone exerts limited antitumor efficacy. $\beta$-elemene, a component of a traditional Chinese medicinal herb, exerts broadspectrum antitumor effects by inhibiting proliferation and inducing apoptosis in several types of solid tumor cells. Although bevacizumab and $\beta$-elemene are effective antitumor agents for the treatment of colorectal cancer and possess low toxicity, their antitumor activities are limited when used alone. Therefore, a combination therapy for advanced colorectal cancer patients who cannot tolerate conventional chemotherapy regimens or who have entered the maintenance treatment stage is likely to be valuable in the clinic. In this study, we evaluated the effects of bevacizumab in combination with $\beta$-elemene in a nude mouse model with HCT-116 cell-derived tumors, and elucidated the related mechanisms underlying the antitumor effects by conducting in vivo experiments. The rationale for this study is as follows: first, $\beta$-elemene has certain cytotoxic effects, which can compensate for inability of bevacizumab to directly kill tumor cells. Second, the curative effect of $\beta$-elemene or bevacizumab alone is limited, and the combination of two drugs may further improve the curative effect. Third, both drugs offer advantages by having low toxicity, relieving clinical symptoms and improving the quality of life, which is consistent with the goals of advanced colorectal cancer treatment. Bevacizumab in combination with $\beta$-elemene is a potential strategy for the establishment of palliative treatment and maintenance treatment regimens for advanced colorectal cancer patients.

\section{Methods}

\section{Cell line and experimental animals}

The HCT-116 human colon cancer cell line was purchased from the Chinese Academy of Sciences (Shanghai, China). $\mathrm{BALB} / \mathrm{c}$ nude mice (4- to 6-week-old, 14-18 g, equal numbers of males and females), were purchased from Huafukang Bioscience Company (Beijing, China). The mice were fed heat-sterilized food and water, and maintained on sterilized bedding in a specific pathogen-free (SPF) laminar flow cabinet with constant temperature $\left(22-25^{\circ} \mathrm{C}\right)$ and constant humidity (40-70\%).

\section{Drugs and reagents}

$\beta$-elemene injections were purchased from Jingang Pharmaceutical (Dalian, China). RPMI-1640 culture medium, PBS, penicillin and streptomycin were purchased from HyClone (USA). Trypsin $(0.25 \%)$ was purchased from Gibco (USA). Fetal bovine serum was purchased from SERANA (Germany). Dimethyl sulfoxide was purchased from Sigma (USA), and 4\% paraformaldehyde fixative solution (P0099) was purchased from Beyotime (China). Rabbit anti-CD31 antibody (bs-0468R) and rabbit antiki-67 antibody (bs2130-R) were purchased from Bioss (China). Rabbit anti-VEGF antibody (BA0407) was purchased from Boster (China). Rabbit anti-bcl-2 antibody (ab182858) was purchased from Abcam (USA). The UltraSensitive $^{\mathrm{TM}}$ SP Rabbit IHC Kit (KIT-9706) and stable DAB chromogenic reagent kit (DAB-2031) were purchased from MXB (China).

\section{Cell culture and animal model}

HCT-116 cells were cultured in RPMI-1640 culture medium containing $10 \%$ fetal bovine serum and $100 \mathrm{U} / \mathrm{mL}$ penicillin and streptomycin in an incubator at $37^{\circ} \mathrm{C}$ under a humidified atmosphere with $5 \% \mathrm{CO}_{2}$ and $95 \%$ air. The culture medium was changed once every two days. Cells were subcultured until they reached $70-80 \%$ confluence. HCT-116 cells in the logarithmic growth phase were selected and suspended in PBS after $0.25 \%$ pancreatic enzyme digestion (cell concentration: $1 \times 10^{7} / \mathrm{mL}$, living cells $>95 \%$ after trypan blue staining). Nude mice were intraperitoneally injected with $0.2 \mathrm{~mL}$ of the cell suspension (total, $2 \times 10^{6}$ cells $/$ mice) in the right axilla. The mice were maintained in the original SPF feeding chamber and observed daily. We measured the tumor size when right axillary subcutaneous plaques became visible to the naked eye. Tumor volume was calculated as follows: $\mathrm{V}=\mathrm{L} \times \mathrm{W}^{2}(\mathrm{~L}$ is the long diameter and $\mathrm{W}$ is the short diameter). When the tumor volume reached approximately $75-100 \mathrm{~mm}^{3}$, implantation was considered successful. We eliminated mice in which the tumors were too small or too large to ensure that the remaining mice were up to the standards required for the experiment. 
Table 1 Groups and drug dose in vivo

\begin{tabular}{lcccc}
\hline Groups & Number & Drug & Dose $(\mathrm{mg} / \mathrm{kg})$ & Time interval \\
\hline Control group & 7 & NS & - & Days 1-12 \\
$\beta$-elemene group & 7 & $\beta$-elemene & 75 & Days 1-12 \\
Bevacizumab group & 7 & Bevacizumab & 5 & Days 1, 4, 7, and 10 (other days: NS) \\
Combination group & 7 & $\beta$-elemene & 75 & Days 1-12 \\
& & Bevacizumab & 5 & Days 1, 4, 7, 10 \\
\hline
\end{tabular}

NS, normal saline.

\section{Dosing method and the experimental animal group}

The experiment was approved by the animal ethics committee of Shengjing Hospital (approval number: 2016ps329k). Dose selection was based on clinical dose and related literature $(18,19)$. In a previous experiment, we used $\beta$-elemene at 50,75 and $100 \mathrm{mg} / \mathrm{kg}$ and bevacizumab at $2.5,5$ and $7.5 \mathrm{mg} / \mathrm{kg}$. According to the tumor inhibition rate and the health of nude mice, we chose $75 \mathrm{mg} / \mathrm{kg}$ as the dose for $\beta$-elemene and $5 \mathrm{mg} / \mathrm{kg}$ for bevacizumab. In the combination group, we used the optimal dose of each drug. The dosage in the experiment, which was intraperitoneal injection of $0.4 \mathrm{~mL} /$ mouse, was consistent with clinical dosage. All drugs were freshly prepared just before use following sterile principles. We randomly divided 28 nude mice into the following four groups, and assigned them numbers using the tail labeling method: control group, $\beta$-elemene group, bevacizumab group and combination group. The groups were treated as follows: control group, received $0.4 \mathrm{~mL}$ physiological saline as a blank control on days $1-12 ; \beta$-elemene group, received $\beta$-elemene at $75 \mathrm{mg} / \mathrm{kg}$ on days 1-12; bevacizumab group, received bevacizumab at $5 \mathrm{mg} / \mathrm{kg}$ on days $1,4,7$, and 10 ; and combination group, received $\beta$-elemene at $75 \mathrm{mg} / \mathrm{kg}$ in days $1-12$ and bevacizumab at $5 \mathrm{mg} / \mathrm{kg}$ on days $1,4,7$, and 10 (Table 1). The mice were euthanized on the $2^{\text {nd }}$ day after the last drug administration, which was the $13^{\text {th }}$ day after the first administration.

\section{Observation of the general status and xenograft volume in nude mice}

During the experiment, we observed and recorded the status of nude mice daily along with the changes in the number of nude mice and in the appearance of the subcutaneous xenografts. The longest and shortest diameters were measured every three days using a Vernier caliper to calculate the tumor volume as follows: $\mathrm{V}=\mathrm{L} \times \mathrm{W}^{2}(\mathrm{~L}$ is the long diameter and $\mathrm{W}$ is the short diameter). With the treatment time on the $\mathrm{X}$-axis, and the tumor volume on the Y-axis, tumor growth curves were drawn. We anesthetized mice using isoflurane and euthanized mice using carbon dioxide asphyxiation on the $2^{\text {nd }}$ day after the last administration, after which we excised tumors, measured the tumor volume and calculated the tumor inhibition rate using the following formula: tumor inhibition rate $=(1-$ average tumor volume of the experimental group average tumor volume of the control group) $\times 100 \%$. To evaluate the effects of the drugs in combination, we calculated the coefficient of drug interaction (CDI) using the formula $C D I=A B /(A \times B)$, where $A B$ is the tumor volume ratio of the combination group to the control group, and $\mathrm{A}$ and $\mathrm{B}$ are the tumor volume ratios of the single agent groups to the control group. A CDI value $<1,=1$ or $>1$ indicates synergistic, additive or antagonistic effects, respectively, which a CDI $<0.7$ indicating a significant synergistic effect (20).

\section{Hematoxylin and eosin (HE) staining}

New fresh tissue specimens were immediately fixed for 12 hours with $4 \%$ paraformaldehyde fixative solution, dehydrated in a graded ethanol series, hyalinized using dimethylbenzene, embedded in paraffin, serially sliced into $4-\mu \mathrm{m}$-thick sections and stained with HE. We observed the histomorphological characteristics of tumor tissues and tumor infiltration under a microscope after HE staining.

\section{Immunohistochemical analysis of ki-67, CD31, VEGF, and $b c l-2$}

Tissue sections were dewaxed in xylene, rehydrated with decreasing concentrations of ethanol, washed in PBS, 


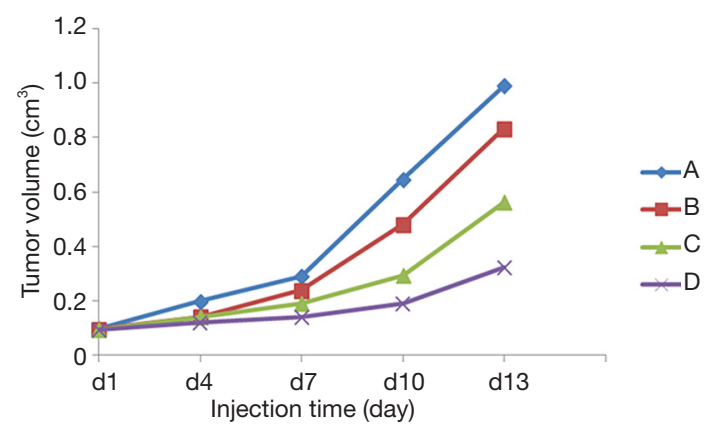

Figure 1 Tumor volume growth curve shows that tumor growth in the three experimental groups was slower than that in the control group, and the tumors in the combination group had the slowest growth. A, control group; B, $\beta$-elemene group; $\mathrm{C}$, bevacizumab group; D, combination group.

incubated with endogenous peroxidase inhibitor and then incubated for 10 minutes in a constant temperature box. The sections were washed using PBS, placed in citrate antigen retrieval solution, and maintained for 5 minutes after they were heated to boiling temperatures. Heating was stopped for 10 minutes and resumed to boiling for 1 minute, and stopped again for 10 minutes. Subsequently, the sections were cooled to room temperature and washed. Serum blocking solution was added and incubated on the sections for 20 minutes at room temperature. Then, antibodies (anti-ki-67 at a dilution of 1:400, anti-CD31 at a dilution of 1:500, anti-VEGF at a dilution of 1:400, and anti-bcl-2 at a dilution of 1:250) were added and incubated for 1 hour at room temperature and at $4{ }^{\circ} \mathrm{C}$ in a humid box overnight. The next day, the sections were removed, incubated for 20 minutes at room temperature, incubated for 20 minutes with a secondary antibody conjugated to biotin, washed, incubated with streptavidin solution, incubated for 20 minutes, washed, and developed with $\mathrm{DAB}$ chromogenic agent. Chromogenic labeling was observed under a microscope, and the sections were counterstained with hematoxylin rinsed, dehydrated and finally sealed off.

For immunohistochemically stained sections, ki67 was expressed in the nucleus. We randomly selected 5 tumor areas at $400 \times$ magnification and counted at least 1,000 tumor cells per group under the microscope. The percentage of positively stained tumor cells was defined as the ki-67 proliferation index. CD31 is expressed in the cytoplasm and on the membrane of vascular endothelial cells, and monoclonal antibodies against CD31 can be used to label tumor microvessels. Positive expression of CD31 was used to calculate the microvessel density (MVD). With reference to the Weidner method (21), we observed all fields at 100x magnification, selected high density areas to look for tumor vessels, and then counted the number of blood vessels stained brown in three fields at $400 \times$ magnification. Any stained tubular structure or endothelial cell cluster was counted as one microvessel as long as the structure remained unlinked. The quantitation was performed in triplicate, and the average value was considered the MVD. VEGF and bcl-2 were both expressed in the cytoplasm of tumor cells. We observed all fields at 100x magnification, selected areas with highly positively staining and randomly counted three fields at $400 \times$ magnification. The percentage of positively stained cells was used as the positive rate of VEGF or bcl-2 expression. The immunohistochemistry results were independently recorded in a blinded manner by a pathologist.

\section{Statistical analyses}

All data are presented as the mean \pm standard deviation (SD). SPSS 17.0 statistical software was used for data analysis. One-way analysis of variance (ANOVA) was used for comparison among groups. A $\mathrm{P}<0.05$ was considered to indicate a statistically significant result.

\section{Results}

\section{General status of and xenograft volume in nude mice}

On the $3^{\text {rd }}$ day after inoculation of HCT-116 cells, most of the nude mice had subcutaneous tumors approximately the size of a rice grain in the right axillary area. All of the nude mice had transplanted tumors of $100 \mathrm{~mm}^{3}$ volume on the $7^{\text {th }}$ day, and the tumor formation rate was $100 \%$. The transplanted tumors grew quickly (Figure 1). The tumors in the control group had the fastest growth, and the symptoms exhibited in these mice were the most serious among the four groups. The tumors in the combination group had the slowest growth, while the growth rates of tumors in the $\beta$-elemene group and bevacizumab group were between those in the two above mentioned groups. Three mice in the combination group exhibited mild diarrhea on the $7^{\text {th }}$ day after inoculation, but these symptoms disappeared automatically on the $9^{\text {th }}$ day. The general status of mice in the bevacizumab group was the best, and that of mice in the control group was the worst, with the mice appearing emaciated. The mice in the control group showed normal food consumption and water intake 

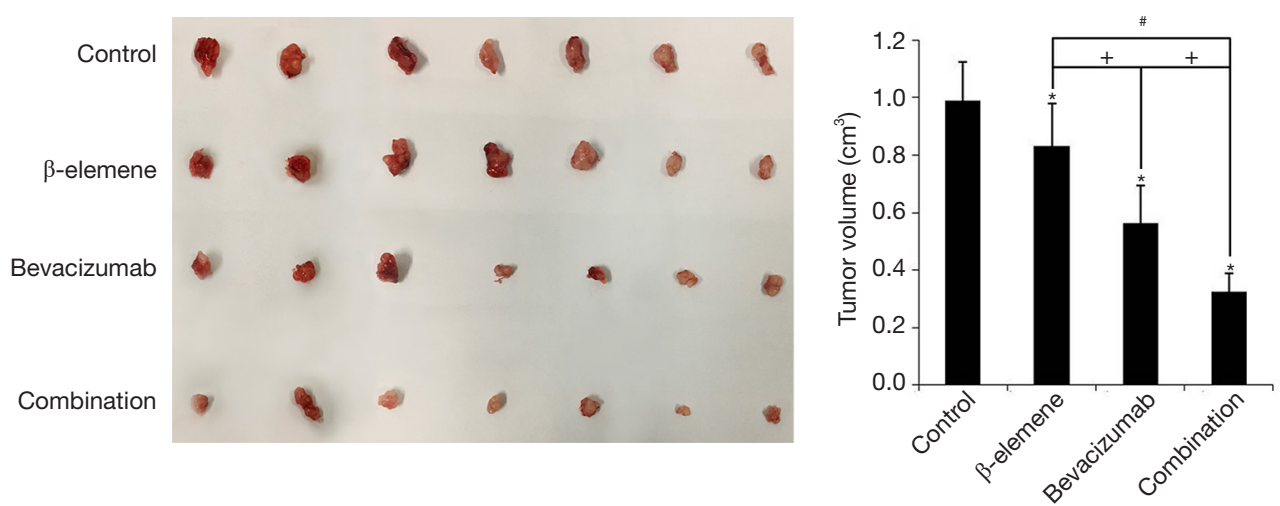

Figure 2 Subcutaneous tumor images and analysis of tumor size of nude mice transplanted with HCT-116 cells in each group. * $\mathrm{P}<0.05$ vs. control group; ${ }^{\prime}, \mathrm{P}<0.05$ vs. $\beta$-elemene group;,$+ \mathrm{P}<0.05$ vs. bevacizumab group.

during the experiment and treatments, the transplantation tumor sizes did not exceed $10 \%$ of the mice's body weight, and the body temperatures were not lower than the normal range. The vital signs of all the mice were stable. Therefore, no one animal had to be sacrificed early due to reaching a humane endpoint.

\section{Comparison of xenograft volume and CDI}

The xenograft volumes in the control, $\beta$-elemene, bevacizumab and combination groups were $0.991 \pm 0.131$, $0.833 \pm 0.145,0.563 \pm 0.131$ and $0.324 \pm 0.066 \mathrm{~cm}^{3}$, respectively (Figure 2). After treatment, the tumors in the three experimental groups were significantly smaller than the tumors in the control group $(\mathrm{P}<0.05)$, and the degree of tumor reduction in the combination group was the most significant (Figure 2). The tumor inhibition rates of the $\beta$-elemene, bevacizumab and combination groups were $15.9 \%, 43.2 \%$ and $67.3 \%$, respectively. The CDI was 0.697 , which indicated that $\beta$-elemene exerted a significant synergistic antitumor effect with bevacizumab.

\section{Pathological and morphological features of tumor tissues}

After HE staining, we observed the tissue sections under the microscope. Tumor cells in the control group appeared abundant and disordered, with varying cell sizes and morphologies; existed in various mitotic phases; and presented large and dense nuclei; furthermore, there were few necrotic areas, and abundant blood vessels (Figure 3). In the three experimental groups, we observed visible diffuse pathological changes in which the tumor cells were relatively sparse with more necrotic areas. We observed karyopyknosis, karyolysis, karyoclasis, and cavity formation within tumor cells and scattered and abundant necrotic foci. The above differences in the features were the most significant in the combination group, as shown in Figure 3.

\section{Effect on tumor cell proliferation in each group}

Immunohistochemistry showed that ki-67 was expressed in the tumor cell nuclei, as indicated by the brown staining. The rate of positive ki-67 expression, or the ki-67 proliferation index, was $30.06 \% \pm 3.37 \%, 19.79 \% \pm 3.75 \%$, $27.51 \% \pm 2.16 \%$ and $16.9 \% \pm 1.09 \%$ in the four groups, respectively. Compared with the control group, ki67 proliferation index of the three experimental groups decreased to different degrees $(\mathrm{P}<0.05)$, among which the combination group had the lowest index, followed by the $\beta$-elemene group. The ki-67 proliferation index of the combination group was lower than that of the $\beta$-elemene group and bevacizumab group $(\mathrm{P}<0.05)$, but the difference between the combination group and the $\beta$-elemene group was not statistically significant (Figure $4 A$ ).

\section{Effect of treatments on tumor angiogenesis}

Microscopic observation showed that the CD31-positive staining was yellow-brown and localized to in the cytoplasm of vascular endothelial cells. The MVDs in the four groups were $45.86 \pm 9.28,31.14 \pm 5.58,27.57 \pm 6.11$ and $12.14 \pm 3.53$, respectively. Compared with the MVD in the control group, the MVDs in each experimental group were reduced to different extents $(\mathrm{P}<0.05)$, with the combination group 


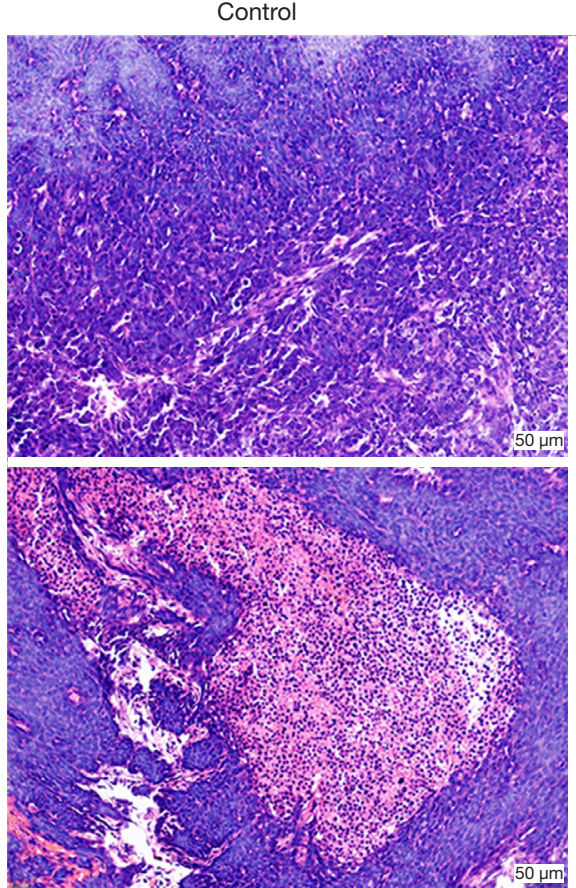

Bevacizumab
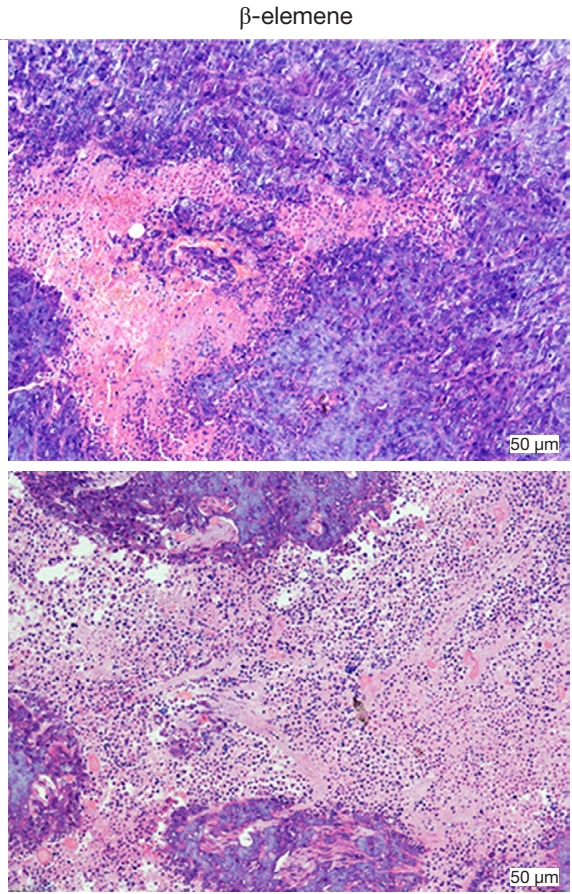

Combination

Figure 3 After HE staining, the tumor cell morphology of xenografts from each group reflects different degrees of necrosis, karyopyknosis, karyolysis, karyoclasis, and cavity formation $(\times 200)$. The above-mentioned changes were the most significant in the combination group. HE, hematoxylin and eosin.

presenting the lowest MVD, followed by the bevacizumab group. The MVD of the combination group was lower than that of the $\beta$-elemene group and bevacizumab group $(\mathrm{P}<0.05)$, but the difference between the $\beta$-elemene group and bevacizumab group was not statistically significant (Figure $4 B$ ).

As shown in Figure 4C, VEGF was expressed on the tumor cell membrane and cytoplasm and stained brown. The positive expression rates of VEGF in the four groups were $39.48 \% \pm 6.72 \%, 28.86 \% \pm 3.54 \%, 25.04 \% \pm 2.64 \%$ and $19.5 \% \pm 2.68 \%$, respectively. Compared with the control group, the three experimental groups showed reduced VEGF expression to different degrees $(\mathrm{P}<0.05)$, with the combination group exhibiting the lowest expression of VEGF, followed by the bevacizumab group. VEGF expression in the combination group was lower than that in the $\beta$-elemene and bevacizumab groups $(\mathrm{P}<0.05)$, but there was no significant difference between the $\beta$-elemene and bevacizumab groups (Figure $4 C$ ).

\section{Effect of treatments on tumor apoptosis}

Immunohistochemistry showed that bcl-2 was expressed in the cytoplasm of tumor cells and stained brown. The positive expression rates of bcl-2 in the four groups were $33.95 \% \pm 6.38 \%, 20.11 \% \pm 9.49 \%, 24.28 \% \pm 6.57 \%$ and $11.18 \% \pm 2.72 \%$, respectively. Compared with the control group, the three experimental groups showed reduced bcl-2 expression to varying degrees $(\mathrm{P}<0.05)$, with the combination group exhibiting the lowest bcl-2 expression, followed by the $\beta$-elemene group. Immunohistochemical staining showed that bcl-2 expression in the combination group was lower than that in the $\beta$-elemene and bevacizumab groups $(\mathrm{P}<0.05)$, but there was no significant difference between the $\beta$-elemene group and bevacizumab group (Figure 4D). Male and female mice in each group showed no differences in any of the experiment.

\section{Discussion}

Colorectal cancer is a common malignancy of the digestive system, with approximately 1.2 million new cases reported in the world annually; approximately $25 \%$ of these cases are accompanied with liver metastasis, and another $25 \%$ of cases are accompanied with subsequent distant 

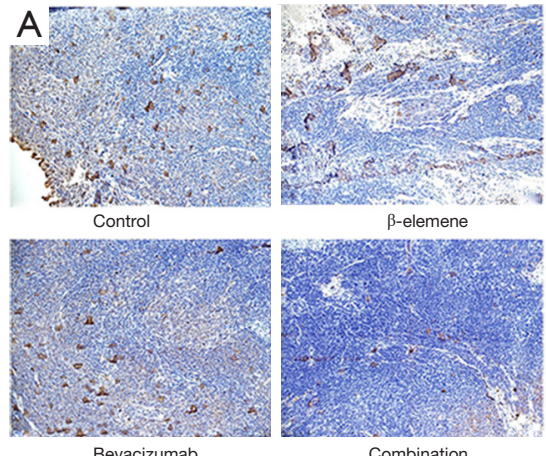

Bevacizumab
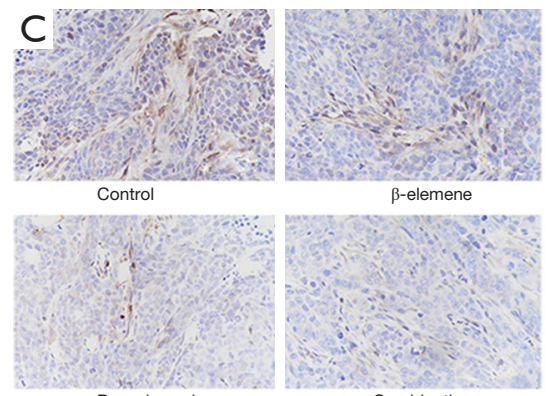

Bevacizumab
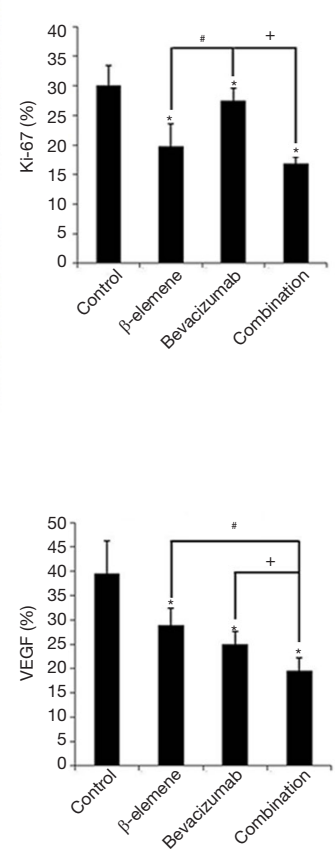

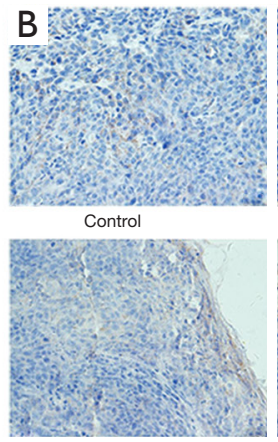

Bevacizumab

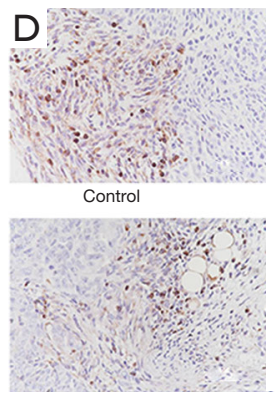

Bevacizumab

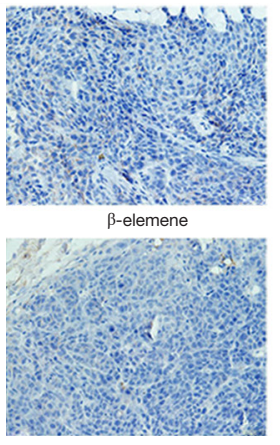

Combination

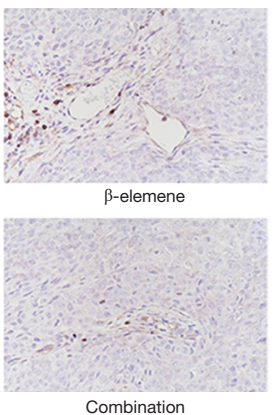

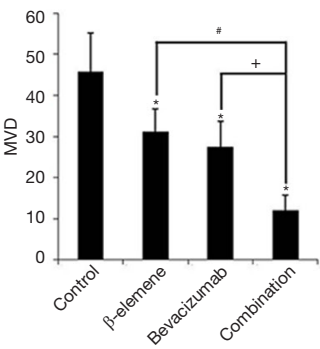

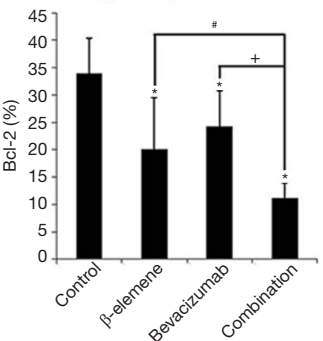

Figure 4 Ki-67, CD-31, VEGF and bcl-2 expression level were downregulated in the three experimental groups and expression levels in the combination group were the lowest for all the proteins tested. (A) Immunohistochemistry images (left panel) and analyses (right panel) of ki67 protein expression in each group, ×200 magnification; (B) immunohistochemistry images (left panel) and analyses (right panel) of CD31 protein expression in the four groups, $\times 400$ magnification; (C) immunohistochemistry images (left panel) and analyses (right panel) of VEGF protein expression in the four groups, $\times 400$ magnification; (D) immunohistochemistry images (left panel) and analyses (right panel) of bcl-2 protein expression in the four groups, $\times 400$ magnification. * $\mathrm{P}<0.05$ vs. control group; ${ }^{*}, \mathrm{P}<0.05$ vs. $\beta$-elemene group;,$+ \mathrm{P}<0.05$ vs. bevacizumab group. VEGF, vascular endothelial growth factor; MVD, microvessel density.

metastasis (22). For these patients with unresectable advanced colorectal cancer, comprehensive treatment and individualized treatment are often needed, including palliative chemotherapy, molecular targeted therapy, and traditional Chinese medicine. Among these therapeutic options, palliative chemotherapy is the most commonly used to improve clinical symptoms, improve quality of life and prolong survival time. Fluorouracil combined with oxaliplatin or irinotecan has been recommended as the first- or second-line standard chemotherapy regimen for advanced colorectal cancer patients (2). However, due to its lack of specificity and dose-limiting toxicity, chemotherapy is sometimes restricted. Bevacizumab is characterized by its low resistance and low toxicity (23). Nevertheless, the objective response rate of bevacizumab alone is limited, as it has a weak cytotoxic effect on tumor cells. Tumor angiogenesis is induced and driven by tumor cells, which secrete VEGF and other angiogenic factors. It is difficult to control tumor neovascularization and tumor progression if the tumor cells cannot be killed effectively. Therefore, bevacizumab needs to be combined with other drugs to effectively inhibit and kill tumor cells.

$\beta$-elemene plays an antitumor role by directly killing tumor cells, enhancing immunity, inducing tumor cell apoptosis, and exerting antiangiogenic effects, among others. $\beta$-elemene possesses certain characteristics, such as a definite antitumor efficacy, a broad antitumor spectrum, low toxicity and reduced side effects, and is widely used for the clinical treatment of liver cancer, lung cancer, gastrointestinal cancer, brain cancer and other solid tumors (24-27). In this study, $\beta$-elemene downregulated ki-67 protein expression to inhibit tumor cell proliferation, downregulated bcl-2 expression to induce apoptosis, and reduced MVD and expression of VEGF to inhibit tumor angiogenesis, which were consistent with the results of previous studies. 
$\beta$-elemene kills tumor cells mainly by inhibiting proliferation and promoting apoptosis (25). Its antiangiogenic potency is relatively weak. Bevacizumab indirectly inhibits tumor growth and metastasis by inhibiting tumor angiogenesis, and its ability to directly kill tumor cells is relatively weak. Accordingly, we used the above two drugs in combination to target different pathways and inhibit tumor growth more effectively. In this study, compared with that in the control group, tumor growth in three experimental groups was reduced to different degrees, and the tumor growth curve in the combination group was the least steep. The combination of bevacizumab with $\beta$-elemene resulted in a more significant reduction in xenograft volume and a greater tumor inhibition rate than those of either bevacizumab or $\beta$-elemene alone. The CDI was 0.697 , which indicated that $\beta$-elemene exerted a significant synergistic antitumor effect with bevacizumab. HE staining showed that tumor cells in the three experimental groups displayed signs of degeneration with different degrees of necrosis, with the combination group showing the most significant increase in necrosis. Compared with those in the control group, the expression levels of ki67, CD31, VEGF and bcl-2 protein were downregulated to different degrees in the three experimental groups, with the combination group displaying the most significant change in expression.

Regarding the inhibition of tumor cell proliferation, a greater reduction in ki-67 protein expression in HCT116 cells was observed in the combination group than in the bevacizumab group. Ki-67 is a nuclear antigen that is closely related to cell proliferation, and its expression level reflects proliferative activity and can be used to determine the rate of growth of malignant tumors (28). Ki67 is mainly expressed in the nucleus, and its expression is highly correlated with the cell cycle. Its expression begins to manifest during the late $G_{1}$ phase, rises gradually during $S$ and $G_{2}$ phases, peaks during $M$ phase, and finally rapidly disappears after mitosis; additionally, ki-67 is not expressed during $G_{0}$ phase (29). The half-life of ki-67 is short and not easily altered by growth factors. These characteristics allow ki-67 to more accurately assess tumor cell proliferation than other indicators of cell proliferation. In this study, downregulation of ki-67 expression was the most significant in the combination group, followed by that in the $\beta$-elemene group. Furthermore, the expression levels of ki-67 in the $\beta$-elemene group and the combination group were lower than those in the bevacizumab group $(\mathrm{P}<0.05)$. The ki-67 proliferation index in the combination group was also lower than that in the $\beta$-elemene group, though the difference was not significant $(\mathrm{P}=0.311)$. These results showed that $\beta$-elemene, bevacizumab and both drugs in combination can inhibit tumor cell proliferation, and the inhibitory effect of the combined treatment was more significant than the effect of bevacizumab alone. Compared with the $\beta$-elemene group, the combination group did not display a significant difference but showed a downward trend in the ki-67 proliferation index. Therefore, combination treatment can further inhibit tumor cell proliferation by downregulating ki-67, resulting in a more significant reduction in tumor size than that achieved by monotherapy.

With regard to the inhibition of tumor angiogenesis, the results of the present study indicated that the combination of bevacizumab and $\beta$-elemene resulted in a more significant reduction in VEGF and CD31 protein expression in HCT116 cells compared to the reduction caused by either bevacizumab or $\beta$-elemene alone. VEGF plays an essential role in promoting tumor angiogenesis by binding VEGFR and activating the VEGF pathway to promote tumor growth and metastasis. Studies have shown that VEGF and its receptors are overexpressed in solid tumors and that VEGF is persistently expressed during tumor initiation and development. Thus, inhibiting the VEGF pathway can affect tumor angiogenesis, restrict the growth of tumors and prevent tumor cell metastasis (30,31). MVD is an index used to detect tumor angiogenesis and can be used to measure the extent of tumor angiogenesis, assess tumor development, and provide a reference for estimating tumor growth, metastasis and prognosis. CD31 is a frequently used marker of vascular endothelial cells and can be used to label capillaries in tumor tissues. In this study, the MVD and VEGF expression were the lowest in the combination group, followed by those in the bevacizumab group. The difference between the combination group and single agent groups was statistically significant. Thus, $\beta$-elemene and bevacizumab can both downregulate CD31 and VEGF expression, and the antiangiogenic ability of bevacizumab is stronger than that of $\beta$-elemene. The combination of the two drugs can further inhibit tumor angiogenesis relative to each drug alone, thereby inhibiting tumor growth more effectively. Therefore, bevacizumab may synergistically inhibit tumor angiogenesis with $\beta$-elemene by downregulating VEGF and CD31, resulting in further tumor shrinkage.

In terms of inducing tumor cell apoptosis, the combination of bevacizumab and $\beta$-elemene caused a more significant reduction in bcl-2 protein expression in HCT116 cells compared to the expression observed in response 
to either drug alone. Tumor cells can undergo necrosis and apoptosis, which are two main cell death mechanisms, and the induction of tumor cell apoptosis is considered more important than the inhibition of excessive tumor cell proliferation during tumor development. Apoptosis is a process of cellular suicide, which is controlled by various genes. The bcl-2 family plays an important role in apoptosis, and bcl-2 is an antiapoptosis gene. In this study, the decrease in bcl-2 expression was the most significant in the combination group, followed by that in the $\beta$-elemene group. The difference between the combination group and single agent group was statistically significant. Therefore, $\beta$-elemene and bevacizumab can both downregulate bcl-2 expression, and combining the two drugs can further induce tumor cell apoptosis by synergistically downregulating bcl-2.

In conclusion, bevacizumab exerts a synergistic effect with $\beta$-elemene on suppressing the growth of xenografts established from HCT-116 human colon cancer cells, and the related mechanisms may include the following: first, combining bevacizumab with $\beta$-elemene enhances the inhibition of tumor cell proliferation by downregulating ki67 , and $\beta$-elemene plays an important role in this process. Second, the two drugs in combination synergistically inhibit tumor angiogenesis by downregulating the expression of VEGF and CD31, and bevacizumab plays a major role in this process. Third, the combination of the two drugs synergistically promotes tumor apoptosis by downregulating $\mathrm{bcl}-2$, and $\beta$-elemene plays a major role in this process.

Although the reduction in ki-67, VEGF, CD31 and bcl-2 expression was considered to be related to tumor growth reduction in this study, the underlying mechanism remains unclear. Further studies are needed to determine which signaling pathways are activated or inhibited by bevacizumab and $\beta$-elemene to effectively treat colorectal cancer. We also need additional clinical studies to confirm whether the antitumor effect observed in animal models can be translated into clinical use in humans. With indepth studies of the combination of various therapeutic approaches, such as the inhibition of tumor proliferation, suppression of tumor angiogenesis and promotion of tumor apoptosis, the combination of bevacizumab with $\beta$-elemene provides a new direction for the clinical treatment of colorectal cancer. Our study also provides novel insights into the aspects of palliative and maintenance treatment for advanced colorectal cancer patients.

\section{Acknowledgments}

Funding: None.

\section{Footnote}

Conflicts of Interest: All authors have completed the ICMJE uniform disclosure form (available at http://dx.doi. org/10.21037/tcr.2019.12.35). The authors have no conflicts of interest to declare.

Ethical Statement: The authors are accountable for all aspects of the work in ensuring that questions related to the accuracy or integrity of any part of the work are appropriately investigated and resolved. The Ethics Committee of Shengjing Hospital Affiliated to China Medical University (Shengyang, China) approved the research, and the ethics number is 2016ps329k.

Open Access Statement: This is an Open Access article distributed in accordance with the Creative Commons Attribution-NonCommercial-NoDerivs 4.0 International License (CC BY-NC-ND 4.0), which permits the noncommercial replication and distribution of the article with the strict proviso that no changes or edits are made and the original work is properly cited (including links to both the formal publication through the relevant DOI and the license). See: https://creativecommons.org/licenses/by-nc-nd/4.0/.

\section{References}

1. Siegel RL, Miller KD, Fedewa SA, et al. Colorectal cancer statistics, 2017. CA Cancer J Clin 2017;67:177-93.

2. Benson AB, Venook AP, Al-Hawary MM, et al. NCCN guidelines insights: colon cancer, version 2.2018. J Natl Compr Canc Netw 2018;16:359-69.

3. Pavlidis ET, Pavlidis TE. Role of bevacizumab in colorectal cancer growth and its adverse effects: a review. World J Gastroenterol 2013;19:5051-60.

4. Folkman J. Tumor angiogenesis: therapeutic implications. N Engl J Med 1971;285:1182-6.

5. Hicklin DJ, Ellis LM. Role of the vascular endothelial growth factor pathway in tumor growth and angiogenesis. J Clin Oncol 2005;23:1011-27.

6. Shord SS, Bressler LR, Tierney LA, et al. Understanding and managing the possible adverse effects associated with 
bevacizumab. Am J Health Syst Pharm 2009;66:999-1013.

7. Fukumura D, Jain RK. Tumor microvasculature and microenvironment: targets for anti-angiogenesis and normalization. Microvasc Res 2007;74:72-84.

8. Yeung Y, Tebbutt NC. Bevacizumab in colorectal cancer: current and future directions. Expert Rev Anticancer Ther 2012;12:1263-73.

9. Krämer I, Lipp HP. Bevacizumab, a humanized antiangiogenic monoclonal antibody for the treatment of colorectal cancer. J Clin Pharm Ther 2007;32:1-14.

10. Mao Y, Zhang J, Hou L, et al. The effect of beta-elemene on alpha-tubulin polymerization in human hepatoma HepG2 cells. Chin J Cancer Res 2013;25:770-6.

11. Li CL, Chang L, Guo L, et al. $\beta$-elemene induces caspasedependent apoptosis in human glioma cells in vitro through the upregulation of Bax and Fas/ FasL and downregulation of Bcl-2. Asian Pac J Cancer Prev 2014;15:10407-12.

12. Fang $M$, Mei X, Yao $H$, et al. $\beta$-elemene enhances anticancer and anti-metastatic effects of osteosarcoma of ligustrazine in vitro and in vivo. Oncol Lett 2018;15:3957-64.

13. Gong M, Liu Y, Zhang J, et al. $\beta$-elemene inhibits cell proliferation by regulating the expression and activity of topoisomerases I and II $\alpha$ in human hepatocarcinoma HepG-2 cells. Biomed Res Int 2015;2015:153987.

14. Li P, Zhou X, Sun W, et al. Elemene induces apoptosis of human gastric cancer cell line BGC-823 via extracellular signal-regulated kinase (ERK) 1/2 signaling pathway. Med Sci Monit. 2017;23:809-17.

15. Chen Z. A new association test based on Chi-square partition for case-control GWA studies. Genet Epidemiol 2011;35:658-63.

16. Jiang Z, Jacob JA, Loganathachetti DS, et al. $\beta$-elemene: mechanistic studies on cancer cell interaction and its chemosensitization effect. Front Pharmacol 2017;8:105.

17. Zhai B, Zhang N, Han X, et al. Molecular targets of $\beta$-elemene, a herbal extract used in traditional Chinese medicine, and its potential role in cancer therapy: a review. Biomed Pharmacother. 2019;114:108812.

18. Jiang ZY, Qin SK, Yin XJ, et al. Synergistic effects of Endostar combined with $\beta$-elemene on malignant ascites in a mouse model. Exp Ther Med 2012;4:277-84.

19. Tsukihara H, Nakagawa F, Sakamoto K, et al. Efficacy of combination chemotherapy using a novel oral chemotherapeutic agent, TAS-102, together with bevacizumab, cetuximab, or panitumumab on human colorectal cancer xenografts. Oncol Rep 2015;33:2135-42.

20. Lu L, Yang LN, Wang XX, et al. Synergistic cytotoxicity of ampelopsin sodium and carboplatin in human non-small cell lung cancer cell line SPC-A1 by G1 cell cycle arrested. Chin J Integr Med 2017;23:125-31.

21. Weidner N, Semple JP, Welch WR, et al. Tumor angiogenesis and metastasis--correlation in invasive breast carcinoma. N Engl J Med 1991;324:1-8.

22. Takayama T, Miyanishi K, Hayashi T, et al. Colorectal cancer: genetics of development and metastasis. J Gastroenterol 2006;41:185-92.

23. Degirmenci M, Karaca B, Gorumlu G, et al. Efficacy and safety of bevacizumab plus capecitabine and irinotecan regimen for metastatic colorectal cancer. Med Oncol 2010;27:585-91.

24. Chang Z, Gao M, Zhang W, et al. Beta-elemene treatment is associated with improved outcomes of patients with esophageal squamous cell carcinoma. Surg Oncol 2017;26:333-7.

25. Guo Z, Liu Z, Yue H, et al. Beta-elemene increases chemosensitivity to 5 -fluorouracil through downregulating microRNA-191 expression in colorectal carcinoma cells. J Cell Biochem 2018;119:7032-9.

26. Liu Y, Jiang ZY, Zhou YL, et al. $\beta$-elemene regulates endoplasmic reticulum stress to induce the apoptosis of NSCLC cells through PERK/IRE1 $\alpha /$ ATF6 pathway. Biomed Pharmacother 2017;93:490-7.

27. Zhu T, Li X, Luo L, et al. Reversion of malignant phenotypes of human glioblastoma cells by $\beta$-elemene through $\beta$-catenin-mediated regulation of stemness-, differentiation- and epithelial-to-mesenchymal transitionrelated molecules. J Transl Med 2015;13:356.

28. Ganepola GA, Mazziotta RM, Weeresinghe D, et al. Gene expression profiling of primary and metastatic colon cancers identifies a reduced proliferative rate in metastatic tumors. Clin Exp Metastasis 2010;27:1-9.

29. Melling N, Kowitz CM, Simon R, et al. High Ki67 expression is an independent good prognostic marker in colorectal cancer. J Clin Pathol 2016;69:209-14.

30. Kajdaniuk D, Marek B, Borgiel-Marek H, et al. Vascular endothelial growth factor (VEGF) - part 1: in physiology and pathophysiology. Endokrynol Pol 2011;62:444-55.

31. Kajdaniuk D, Marek B, Foltyn W, et al. Vascular endothelial growth factor (VEGF) - part 2: in endocrinology and oncology. Endokrynol Pol 2011;62:456-64.

Cite this article as: Li JN, Xie F, Wang Y. Synergistic effects of bevacizumab in combination with $\beta$-elemene on subcutaneous xenografts derived from HCT-116 human colon cancer cells. Transl Cancer Res 2020;9(2):1001-1011. doi: 10.21037/ tcr.2019.12.35 